\title{
Utilização dos serviços de saúde no Brasil: gênero, características familiares e condição social ${ }^{1}$
}

\author{
Cláudia Travassos, ${ }^{2}$ Francisco Viacava, ${ }^{2}$ Rejane Pinheiro ${ }^{3}$ \\ e Alexandre Brito ${ }^{3}$
}

RESUMO Objetivo. Investigar o perfil de utilização de serviços de saúde por homens e mulheres no Brasil.

Métodos. Utilizaram-se os dados da Pesquisa Nacional por Amostra de Domicílios realizada em 1998. Foram desenvolvidos modelos de regressão logística para analisar as informações relativas a homens e mulheres com e sem restrição de atividades por motivos de saúde nos 15 dias que antecederam a pesquisa. As variáveis individuais foram: posição no mercado de trabalho, escolaridade e raça. Também foram analisadas variáveis relativas à família: renda familiar per capita, tamanho da família e escolaridade e posição do chefe da família no mercado de trabalho. Nos modelos em dois níveis (variáveis individuais e familiares) evidenciouse um efeito de família que, entretanto, não foi captado pelas variáveis analisadas.

Resultados. As mulheres utilizaram mais os serviços de saúde, mesmo quando controlado o efeito da restrição de atividades por motivo de saúde. O uso de serviços de saúde por homens e mulheres dependeu do poder aquisitivo das famílias e das características sociais do próprio indivíduo, definindo um perfil de desigualdades sociais. Observaram-se diferenças no perfil de desigualdade nos gêneros entre pessoas que referiram e não referiram restrição de atividades. As variáveis familiares tiveram maior importância na explicação do consumo de serviços por pessoas sem restrição de atividade.

Conclusões. A formulação de políticas voltadas para a redução das desigualdades no consumo de serviços de saúde deve considerar as diferenças entre mulheres e homens, além da importância das características familiares. Ressalta-se a necessidade de incluir as dimensões gênero e família na especificação dos modelos de uso de serviços de saúde.

Palavras-chave Acesso aos serviços de saúde, utilização de serviços de saúde.

É bem conhecido o fato de que as mulheres fazem maior uso dos ser-

1 Estudo financiado pela Organização Pan-Americana da Saúde através do Concurso Regional de Investigación 1999 sobre "Género y equidad en el acceso a la atención de la salud en las reformas de los sistemas de salud y seguridad social". HDP/HDR/ RGP/81/5.3.

2 Fundação Oswaldo Cruz, Centro de Informação Científica e Tecnológica, Departamento de Informações em Saúde, Avenida Brasil 4365, Rio de Janeiro, RJ, CEP 21045-900, Brasil. Email:viacava@cict.fiocruz.br

3 Universidade Federal do Rio de Janeiro, Núcleo de Estudos em Saúde. viços de saúde do que os homens, e de que essa diferença é, em grande parte, relacionada a questões reprodutivas (1). Entretanto, as diferenças de gênero relativas ao perfil de morbi-mortalidade não se resumem à saúde reprodutiva. As mulheres tendem a avaliar seu estado de saúde de maneira mais negativa e também referem mais doenças crônicas do que os homens, que, por sua vez, apresentam doenças comparativamente mais severas e de maior letalidade (2). Frente às mudanças ocorridas no papel social das mulheres nas últimas décadas, mais recentemente vários autores vêm questionando a existência de desigualdades entre os gêneros em termos de saúde. Arber e Cooper (3), analisando dados da Inglaterra para os anos de 1992 a 1994, demonstraram que, em idades mais avançadas, homens e mulheres tendem a avaliar de maneira semelhante seu estado de saúde. Porém, 
para um dado nível de limitação física, as mulheres tendem a avaliar mais positivamente seu estado de saúde.

No que se refere à utilização de serviços, verificou-se que, nos Estados Unidos, as mulheres procuram serviços preventivos mais freqüentemente e usam mais medicamentos do que os homens; não foram observadas diferenças importantes de gênero no uso de serviços curativos (4). Do ponto de vista comportamental, a procura por serviços de saúde tem sido explicada pelas diferenças na forma como homens e mulheres expressam a doença, o que por, sua vez, estaria associado aos diferentes papéis sociais que assumem em cada sociedade. Por exemplo, homens e mulheres portadores de doenças crônicas, com papéis sociais semelhantes, diferem pouco no comportamento de procura por serviços de saúde (5).

Além disso, enquanto eixo central de procriação e socialização, as famílias assumem importante papel como espaço criador de oportunidades e de perspectivas de vida para seus membros (6) e, por conseguinte, podem interferir no consumo de serviços de saúde. Schor et al. (7), analisando o uso de serviços ambulatoriais por clientes de um plano de saúde, verificaram que o tamanho da família é um elemento importante no consumo individual. Conforme esses autores, há grande variação no consumo entre famílias, sendo que um número pequeno de famílias dá conta da utilização de uma significativa proporção dos serviços de saúde.

Pode-se dizer que as desigualdades no uso de serviços de saúde refletem não apenas as desigualdades individuais no risco de adoecer e morrer (8, 9), mas, também, as diferenças no comportamento do indivíduo perante a doença (10) e as características da oferta de serviços que cada sociedade disponibiliza para seus membros (11, 12). No Brasil, o acesso aos serviços de saúde públicos e privados, contratados pelo governo, é universal e garantido pela constituição federal de 1988. Entretanto, atualmente, $25 \%$ da população já são cobertos por seguro privado de saúde. Diversas mudanças vêm sendo implantadas na política de assistência à saúde no Brasil ao longo dos últimos 10 anos, que incluem: a) mudança de orientação do arcabouço institucional no sentido da descentralização; b) deslocamento da atenção hospitalar para a esfera ambulatorial (13); c) regulamentação do setor de seguros privados; e d) desenvolvimento de programas de atenção básica. Nesse contexto, tornase importante oferecer subsídios para a formulação de políticas voltadas para a redução das desigualdades na utilização de serviços de saúde.

Avançando nessa direção, este estudo objetivou analisar a importância do gênero como categoria de análise no consumo de serviços de saúde. Além disso, foram analisadas a relação entre gênero e condição social do indivíduo e a influência das características da família no perfil de consumo de serviços de saúde de homens e mulheres no Brasil.

\section{MATERIAIS E MÉTODOS}

Utilizaram-se os dados da Pesquisa Nacional por Amostra de Domicílios (PNAD) realizada em 1998 pela Fundação Instituto Brasileiro de Geografia e Estatística (IBGE) (14). A amostra da PNAD de 1998, constituída por $110 \mathrm{mil}$ domicílios, com informações sobre todos os moradores desses domicílios ( $\mathrm{n}=344$ 975), foi obtida por um processo de amostragem complexo, em multi-estágios. Para garantir a representatividade da amostra, a análise se baseou nos pesos proporcionais às diferentes probabilidades de seleção. Foram utilizados os pesos relativos, obtidos dividindo-se os pesos de expansão pela média dos pesos (15).

Após a análise descritiva, excluíram-se da amostra as crianças menores de 10 anos por não poderem ser classificadas na variável posição no mercado de trabalho. Mesmo assim, as crianças menores de 10 anos foram mantidas para fins de composição das famílias. Excluíram-se também as pessoas residentes nas áreas rurais, em função das particularidades das condições sociais e do padrão diferenciado de oferta e uso de serviços de saúde naquela população.
O uso de serviços de saúde foi medido a partir de resposta afirmativa sobre a utilização de algum tipo de serviço de saúde nos 15 dias que antecederam a entrevista, incluindo farmácia; posto ou centro de saúde; consultório médico particular; consultório odontológico; consultório de profissionais não médicos, como fonoaudiólogos ou psicólogos; ambulatório ou consultório de empresa ou sindicato; ambulatório ou consultório de clínica; pronto-socorro ou emergência; hospital; laboratório ou clínica para exames complementares; atendimento domiciliar; e outros serviços.

A idade, variável de confundimento no estudo de desigualdades sociais no uso de serviços de saúde, foi controlada na análise. A variável de morbidade utilizada foi a restrição de atividades rotineiras por motivo de saúde nos 15 dias que antecederam a entrevista, por ser a que apresentou maior poder explicativo do uso de serviços de saúde quando comparada à referência a doenças crônicas ou autoavaliação do estado de saúde em estudo anterior (16). Em função da força dessa variável de morbidade e da ocorrência de interação entre sexo e morbidade em relação ao uso de serviços de saúde, optou-se por analisar o efeito das variáveis sociais a partir de quatro modelos: homens e mulheres com restrição de atividades rotineiras (uso de serviços curativos) e homens e mulheres sem restrição de atividades rotineiras (uso de serviços preventivos e de acompanhamento).

Analisaram-se os efeitos das características demográficas e sociais e da composição da família no uso de serviços de saúde pelos indivíduos. Os fatores sociais testados foram posição no mercado de trabalho, escolaridade e raça. A posição no mercado de trabalho é uma aproximação da variável sugerida por Arber (17), adaptada para a realidade brasileira, com a criação de 10 categorias que levaram em consideração a inserção ou não do indivíduo no mercado de trabalho: 1) empregados no mercado formal (com carteira de trabalho assinada); 2) empregados no mercado informal (sem carteira de trabalho assinada); 
3) empregadores (com empreendimento próprio e pelo menos um empregado); 4) autônomos (trabalhadores com empreendimento próprio, trabalhando sozinhos ou com sócios, sem empregados, contando ou não com a ajuda de trabalhador não remunerado); 5) autoconsumo (pessoa que trabalha, durante pelo menos 1 hora na semana, em atividades da agricultura, silvicultura, pecuária, extração vegetal, pesca e piscicultura, para alimentação própria e de pelo menos um membro da unidade domiciliar, e trabalhador na construção para uso próprio, ou seja, pessoa que trabalha pelo menos 1 hora na semana na construção de edificações, estradas privativas, poços e outras benfeitorias, exceto as obras destinadas unicamente à reforma, para uso próprio ou de pelo menos um membro da unidade domiciliar); 6) desempregados (que referiram não estar trabalhando, mas ter procurado trabalho na semana de referência); 7) aposentados (pessoas que não estavam trabalhando na semana de referência, que não procuraram trabalho e que recebiam aposentadoria); 8) estudantes (pessoas sem trabalho, sem aposentadoria e que referiram estar freqüentando algum curso); 9) afazeres domésticos (pessoas não classificadas em nenhuma das categorias anteriores e que cuidavam dos afazeres domésticos na semana de referência); 10) outros (não classificados nas outras categorias, como por exemplo pessoas que vivem de renda ou pensão).

A escolaridade foi definida em categorias de séries completas de estudo: sem instrução; fundamental básico (1 a 4 anos de estudo); fundamental (5 a 8 anos); ensino médio (9 a 11 anos) e superior (12 anos ou mais). Para a raça, que na PNAD é definida pelo entrevistado, as informações foram agregadas em duas categorias: pessoas brancas e não brancas. Entre os fatores relacionados à família foram incluídos: renda familiar per capita, tamanho da família e características do chefe (idade, sexo, escolaridade e posição no mercado de trabalho).

A estrutura hierarquizada dos dados em dois níveis - individual e familiar - implicou na utilização de

TABELA 1. Porcentagem de utilização de serviços de saúde segundo sexo, faixa etária e restrição de atividades rotineiras por motivo de saúde, Brasil, 1998a

\begin{tabular}{|c|c|c|c|c|c|c|}
\hline \multirow[b]{2}{*}{ Faixa etária } & \multicolumn{3}{|c|}{ Homens } & \multicolumn{3}{|c|}{ Mulheres } \\
\hline & $\begin{array}{c}\text { Sem } \\
\text { restrição }\end{array}$ & $\begin{array}{c}\text { Com } \\
\text { restrição }\end{array}$ & Total & $\begin{array}{c}\text { Sem } \\
\text { restrição }\end{array}$ & $\begin{array}{l}\text { Com } \\
\text { restrição }\end{array}$ & Total \\
\hline 0 a 4 anos & 13,8 & 66,3 & 17,4 & 13,2 & 66,5 & $16,5^{c}$ \\
\hline 5 a 9 anos & 6,5 & 58,5 & 9,3 & 6,7 & 55,7 & 9,1 \\
\hline 10 a 14 anos & 5,2 & 56,3 & 7,0 & $5,8^{b}$ & 53,0 & 7,5 \\
\hline 15 a 24 anos & 4,8 & 52,3 & 6,3 & $10,4^{b}$ & $59,5^{b}$ & $12,6^{b}$ \\
\hline 25 a 49 anos & 6,2 & 53,8 & 8,5 & $13,7^{b}$ & $61,6^{b}$ & $17,1^{b}$ \\
\hline 50 a 64 anos & 9,4 & 52,2 & 13,3 & $17,5^{\mathrm{b}}$ & $58,4^{b}$ & $22,2^{b}$ \\
\hline 65 ou mais & 13,4 & 53,4 & 18,9 & $17,9^{b}$ & 55,8 & $23,8^{b}$ \\
\hline Total & 7,2 & 55,5 & 9,9 & $12,1^{b}$ & $59,4^{b}$ & $15,4^{b}$ \\
\hline
\end{tabular}

Fonte: Pesquisa Nacional por Amostra de Domicílios (PNAD), 1998.

a Restrição das atividades nos 15 dias que antecederam o estudo.

b $P<0,01$.

c $0,01<P<0,05$.

ferramental analítico que corrige as estimativas da variância resultante da violação do princípio de independência entre as observações. Para corrigir o erro padrão dos coeficientes do modelo foram utilizados modelos hierárquicos clássicos e bayesianos, utilizando-se o programa Mlwin (versão 1.10.0006). Calcularam-se os coeficientes de correlação intra-classe, que expressam a proporção da variabilidade devida ao nível da família em relação à variabilidade total do modelo. Utilizou-se o cálculo proposto por Snijders e Bosker (18) para aplicação em modelos logísticos. Nenhuma característica das famílias foi capaz de explicar a variabilidade observada no uso de serviços de saúde entre as famílias. Os métodos logísticos tradicionais e os métodos clássicos e bayesianos usados na modelagem hierárquica revelaram estimativas semelhantes para os coeficientes fixos $(\beta)$ e seus erros padrão. Assim, o modelo hierárquico pôde ser substituído por modelos de regressão logística tradicionais.

No ajuste dos quatro modelos utilizou-se a razão de verossimilhança (diferença nos valores dedeviance). Assintoticamente, a diferença nos valores de deviance possui distribuição semelhante à distribuição do qui-quadrado $\left(\chi^{2}\right)$ com $m$ graus de liberdade, onde $m$ é a diferença de parâmetro entre os dois modelos (19). Na análise comparativa dos modelos explicativos de uso de serviços de saúde por homens e mulheres, testou-se a diferença entre os valores de deviance $(P=0,05)$ para cada variável em relação ao modelo com todas as variáveis e, posteriormente, adotou-se a abordagem de retirada seqüencial de variáveis para o ajuste do modelo final. Assim, cada variável foi mantida no modelo sempre que sua retirada piorasse de forma estatisticamente significativa o ajuste do modelo. A existência de desigualdades sociais foi também avaliada a partir da razão de chance (odds ratio, OR) com intervalo de confiança de 95\%. Para corrigir o efeito do desenho amostral na estimativa das variâncias, utilizou-se o programa computacional SUDAAN (20).

\section{RESULTADOS}

Considerando-se a população total no Brasil em 1998, as taxas de utilização de serviços de saúde nos 15 dias que antecederam a entrevista foram maiores para as mulheres do que para os homens (tabela 1). Como esperado, as curvas das taxas de utilização para os homens tiveram formato em $U$, com os valores mais baixos em torno da faixa de 15 a 24 anos. Para as mulheres, as curvas de utilização aproximaram-se mais de um J, com os valores mais baixos na faixa etária de 10 a 14 anos. 
TABELA 2. Variância no padrão de uso de serviços de saúde em função da inclusão de variáveis familiares ${ }^{a}$ no modelo de análise e coeficientes de correlação intra-classe para os modelos de homens e mulheres com e sem restrição de atividades, Brasil, 1998

\begin{tabular}{lccccc}
\hline \multirow{2}{*}{$\begin{array}{c}\text { Modelo apenas } \\
\text { com intercepto }\end{array}$} & \multicolumn{2}{c}{ Com restrição de atividades } & & \multicolumn{2}{c}{ Sem restrição de atividades } \\
\cline { 2 - 3 } & Homens & Mulheres & & Homens & Mulheres \\
\hline $\begin{array}{l}\text { Variância (erro padrão) } \\
\begin{array}{l}\text { Coeficiente de correlação } \\
\text { intra-classe }\end{array}\end{array}$ & $0,087(0,075)$ & $0,098(0,061)$ & & $0,633(0,053)$ & $0,522(0,031)$ \\
$\begin{array}{l}\text { a As seguintes características foram levadas em consideração: renda familiar per capita, tamanho da família e características do chefe (idade, sexo, es- } \\
\text { colaridade e posição no mercado de trabalho). }\end{array}$ & 0,026 & 0,029 & 0,160 & 0,140 \\
\hline
\end{tabular}

Para as pessoas que referiram restrição de atividades rotineiras por motivo de saúde, as taxas de utilização foram muito maiores nos dois sexos, mas a taxa de utilização das mulheres permaneceu maior do que a taxa dos homens, sendo que a diferença entre taxas dos dois sexos se reduziu. Os homens com restrição de atividade utilizaram mais serviços de saúde do que as mulheres até os 14 anos; o padrão se inverteu dos 15 aos 64 anos. A partir dos 65 anos, entre pessoas que referiram restrição de atividades, a diferença nas taxas de utilização entre os gêneros tendeu a desaparecer. Para as pessoas que não referiram restrição de atividades rotineiras, o comporta- mento das taxas de utilização se aproximou das taxas totais, já que desse grupo fazem parte a maioria das pessoas da amostra.

O emprego de modelos hierárquicos demonstrou a existência de variabilidade no uso de serviços de saúde associada às características das famílias. O coeficiente de correlação intraclasse (tabela 2), que expressa a proporção da variabilidade devida à inclusão no modelo do nível da família em relação à variabilidade total do modelo, apresentou valores elevados nos modelos de uso de serviços preventivos e de acompanhamento (pessoas sem restrição). No entanto, as características sociais e demográficas das famílias analisadas não diminuíram a variância do nível da família e não auxiliaram na explicação do efeito aleatório da família. Por esta razão, como mencionado nas análises a seguir, foram utilizados modelos de regressão tradicional.

Os quatro modelos de uso de serviços de saúde analisados (tabela 3 ) indicaram um melhor ajuste (medido pela diferença entre os valores de $d e$ viance entre o modelo completo e o modelo apenas com o intercepto) nas pessoas sem restrição do que nas pessoas com restrição de atividades rotineiras em função de problemas de saúde. Da mesma forma, observou-se também um melhor ajuste nos modelos das

TABELA 3. Comparação entre modelos de uso de serviços de saúde, Brasil, 1998

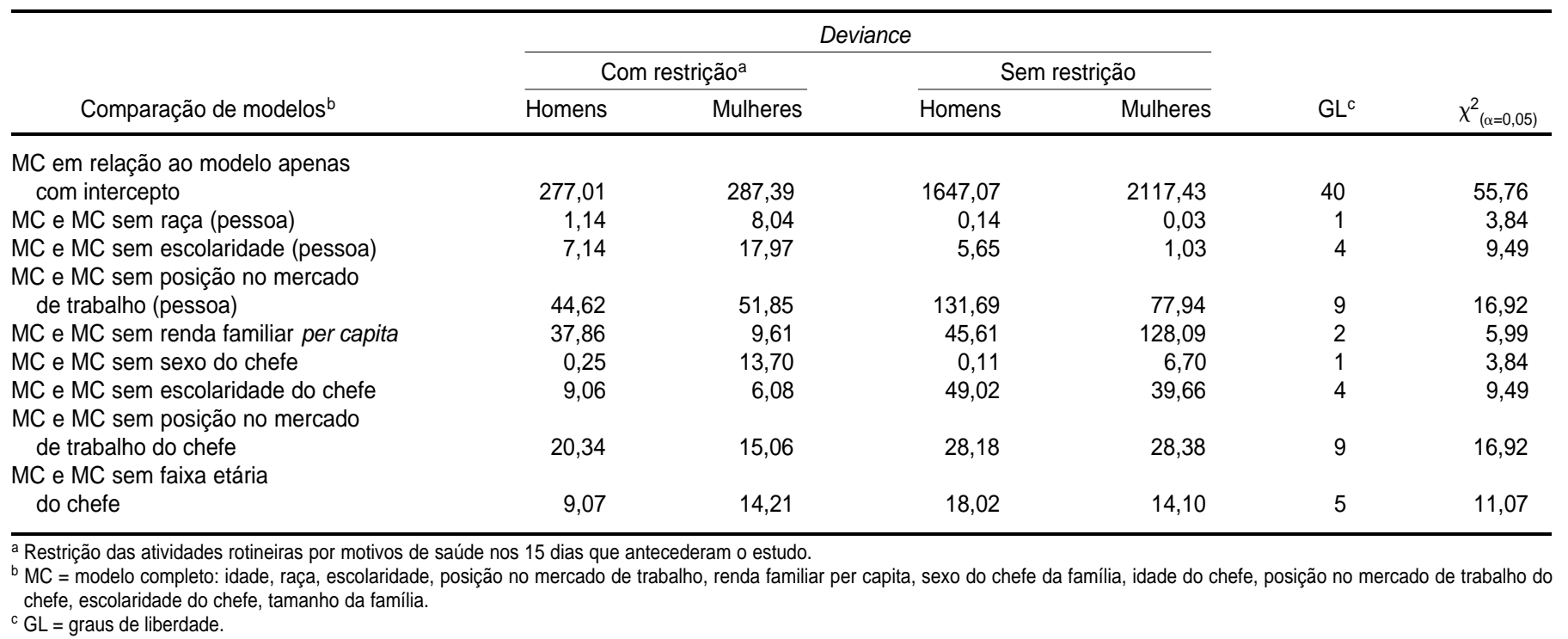


mulheres do que nos modelos dos homens, em ambos os tipos de serviços.

Analisando a contribuição de cada variável para explicar o uso de serviços de saúde com base nos valores de deviance e nos graus de liberdade, é possível inferir que, no caso das pessoas com restrição, após controle da idade, a renda familiar per capita é um fator com grande força explicativa do uso de serviços nos homens, mas seu efeito é bem menor nas mulheres. A posição do indivíduo no mercado de trabalho determina o uso em ambos os sexos, porém, nas mulheres, tem efeito maior do que a renda. A posição do indivíduo no mercado de trabalho, a escolaridade e o sexo do chefe da família são os fatores explicativos mais importantes no caso das mulheres. Por outro lado, raça e escolaridade do indivíduo explicaram o uso de serviços por mulheres que referiram restrição de atividades, mas não pelos homens; e a escolaridade do chefe não se mostrou estatisticamente significativa em nenhum dos sexos. A posição do chefe no mercado de trabalho teve efeito no uso de serviços entre os homens, mas não entre as mulheres. Por fim, a idade do chefe determinou o uso de serviços apenas entre mulheres (tabela 3).

No uso de serviços de saúde entre pessoas sem restrição, a posição no mercado de trabalho do indivíduo e a renda familiar per capita aparecem como as variáveis com maior poder explicativo, tanto para os homens como para as mulheres. Entretanto, a posição no mercado de trabalho é mais importante na determinação do uso de serviços pelos homens e a renda familiar per capita, na determinação do uso pelas mulheres. A escolaridade não foi determinante do uso de serviços em nenhum dos sexos, mas a escolaridade do chefe apareceu como fator explicativo importante. $\mathrm{O}$ sexo do chefe determinou as chances de uso apenas para as mulheres. A posição do chefe no mercado de trabalho teve menor força explicativa do que a posição do próprio indivíduo em ambos os gêneros, mas manteve um papel na determinação do uso. A idade do chefe também contribuiu para explicar o padrão de uso em ambos os sexos. Não se ob- servou efeito de raça nas chances de uso de serviços preventivos e de acompanhamento (tabela 3).

Com relação à idade, as estimativas obtidas nos modelos de regressão, no caso das pessoas com restrição (tabela 4), indicam que essa variável não se associou ao uso de serviços nas mulheres, mas apresentou um efeito importante nos homens. Chama a atenção o fato de que os homens entre 10 e 14 anos são os que apresentaram maior chance de uso; mesmo entre homens com mais de 65 anos a razão de chance foi inferior $(\mathrm{OR}=0,68)$. Nas pessoas sem restrição(tabela 5) infere-se, a partir da análise, que a idade esteve associada à utilização nos homens e nas mulheres. No caso das mulheres, esta associação foi positiva, isto é, as chances de uso aumentaram com a idade as mulheres entre 50 e 64 anos apresentaram chances de uso 2,32 vezes maiores do que as mulheres entre $10 \mathrm{e}$ 14 anos, enquanto que as mulheres com mais de 65 anos apresentaram chances 2,26 vezes maiores. Nos homens, o grupo de 15 a 24 anos apresentou a menor chance de uso $(\mathrm{OR}=$ 0,86); somente após a idade de 50 anos é que as chances de uso foram maiores do que as dos jovens entre 10 e 14 anos $(\mathrm{OR}=1,47$ para homens de 50 a 64 anos, e OR = 1,71 para homens com mais de 64 anos).

O padrão de desigualdades sociais no consumo de pessoas com restrição, em ambos os sexos, tomando como referência as razões de chances, variou nos gêneros. A raça explicou a diferença no uso das mulheres: as mulheres brancas apresentaram maiores chances de uso do que as não brancas $(\mathrm{OR}=1,14)$. Homens e mulheres patrões ou empregados no setor formal da economia apresentaram maiores chances de uso. A desigualdade de renda no uso de serviços de cuidados a pacientes agudos foi maior para os homens $(\mathrm{OR}=1,45$ para homens com renda familiar per capita entre 0,75 e 2 salários mínimos, e OR = 1,90 para homens com renda familiar per capita acima de 2 salários mínimos em relação àqueles cuja renda familiar per capita era menor do que 0,75 salários mínimos) do que para as mulheres
$(\mathrm{OR}=1,14$ para mulheres com renda familiar per capita entre 0,75 e 2 salários mínimos, e OR = 1,27 para mulheres com renda familiar per capita acima de 2 salários mínimos). A escolaridade teve impacto positivo no uso dos serviços pelas mulheres. Mulheres pertencentes a famílias com chefe homem possuíam chances de uso $23 \%$ maiores do que as pertencentes a famílias chefiadas por mulheres.

Em relação às variáveis demográficas da família, o tamanho associou-se ao uso de serviços de cuidados a pacientes agudos (pessoas com restrição) apenas nas mulheres, com as chances de uso diminuindo à medida que a família aumentava. A idade do chefe, apesar de contribuir para o ajuste no modelo de mulheres, não apresentou diferença estatisticamente significativa para nenhuma faixa etária $(P>0,05)$.

Com relação às pessoas sem restrição, observaram-se também iniqüidades em ambos os sexos. Ceteris paribus, as desigualdades associadas à renda foram semelhantes nos dois gêneros, mas as desigualdades em função da escolaridade do chefe foram mais evidentes nos homens. As mulheres de famílias com chefes do sexo masculino apresentaram maiores chances de uso do que aquelas de famílias com chefes mulheres. As aposentadas e donas de casa apresentaram maiores chances de uso ( $\mathrm{OR}=1,26$ e 1,18, respectivamente) do que aquelas inseridas no mercado de trabalho, desempregadas ou estudantes. Entre os homens, os aposentados apresentaram a maior chance de uso $(\mathrm{OR}=1,85)$; os empregados no mercado informal e os que trabalhavam por conta própria apresentaram as menores chances de uso em relação aos empregados no setor formal (OR = 0,81 e 0,77 , respectivamente).

O tamanho da família apareceu bastante associado ao uso de serviços preventivos em ambos os sexos $(P<$ $0,001)$, com as chances de uso diminuindo à medida que a família aumentava. A idade do chefe, apesar de contribuir para o ajuste dos modelos de homens e mulheres, teve efeito pequeno. Destaca-se o fato de as mulheres de famílias com chefes jovens (até 24 anos) apresentarem maior chance 
TABELA 4. Modelos logísticos para uso de serviços de saúde em pessoas que apresentaram restrição de atividades rotineiras em função de problemas de saúde, Brasil, 1998 a,b,c

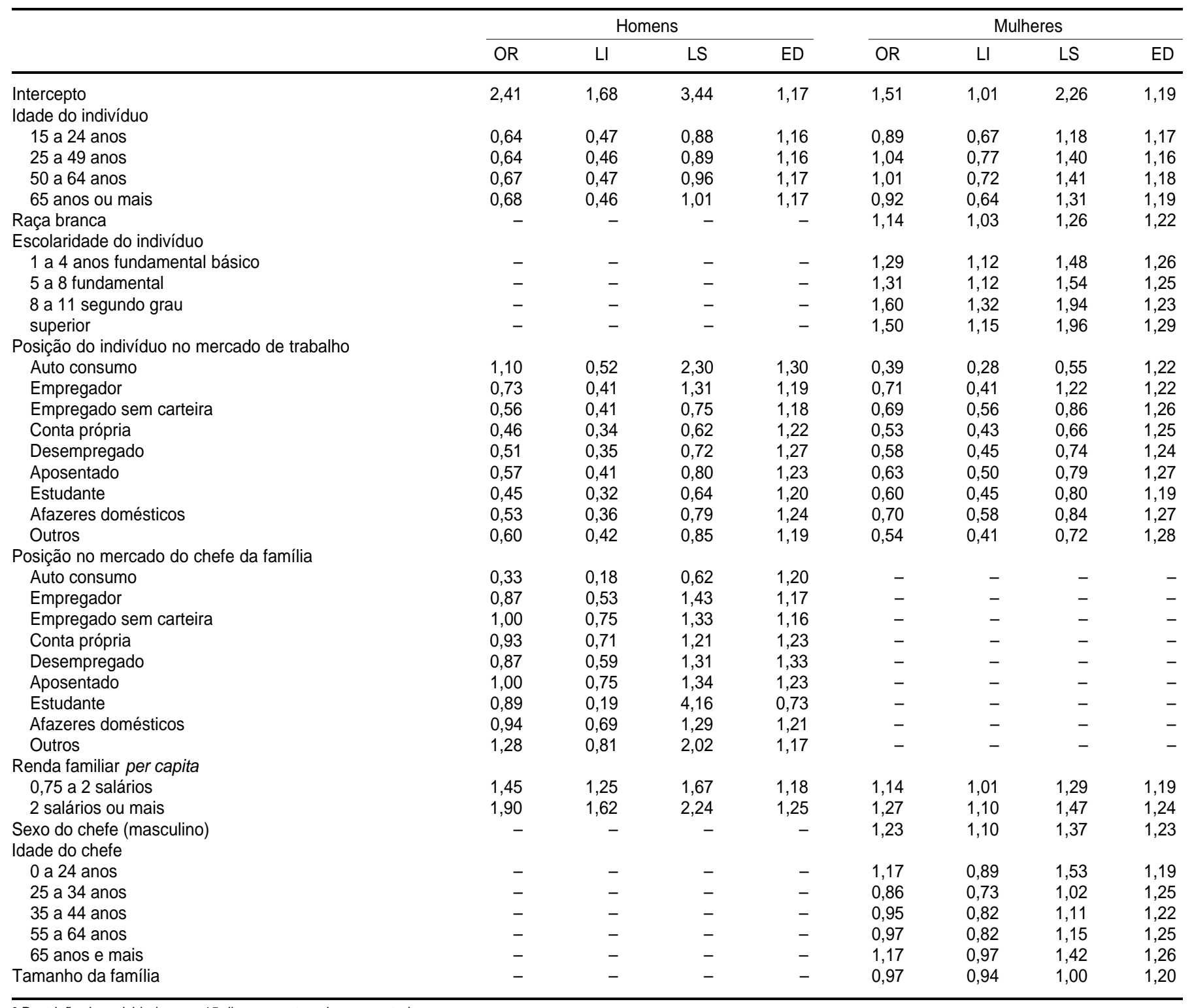

a Restrição das atividades nos 15 dias que antecederam o estudo.

b Categorias de referência: idade de 10 a 14 anos; raça não branca; escolaridade sem instrução; trabalhador com carteira assinada; renda de 0 a 0,75 salário mínimo; sexo feminino para chefe de família; idade do chefe entre 45 e 54 anos.

${ }^{c} \mathrm{OR}=$ razão de chances; $\mathrm{LI}=$ limite inferior; $\mathrm{LS}=$ limite superior; $\mathrm{ED}=$ efeito do desenho amostral.

de uso de serviços preventivos do que aquelas pertencentes a famílias com chefes de maior idade ( $\mathrm{OR}=1,17)$.

\section{DISCUSSÃO}

No caso brasileiro, as mulheres utilizam mais os serviços de saúde do que os homens, padrão que se mantém quando se desagregam os dados pela restrição ou não de atividades por motivo de saúde. Considerando separadamente os homens e mulheres com e sem restrição das atividades por motivos de saúde, foi constatado que as mulheres brancas, com maior escolaridade, maior poder aquisitivo, empregadas no setor formal da economia e pertencentes a famílias chefiadas por homens, apresentaram as maiores chances de uso de serviços de saúde na presença de restrição de atividade por motivo de saúde. Homens de maior poder aquisitivo e ocupando posições de patrão e empregado no setor formal da economia, independentemente da raça, da escolaridade e do sexo do chefe da família, foram os que apresentaram as maiores chances de uso de 
TABELA 5. Modelos logísticos para uso de serviços de saúde em pessoas que não apresentaram restrição de atividades rotineiras ${ }^{\mathrm{a}}$ em função de problemas de saúde, Brasil, 1998b

\begin{tabular}{|c|c|c|c|c|c|c|c|c|}
\hline & \multicolumn{4}{|c|}{ Homens } & \multicolumn{4}{|c|}{ Mulheres } \\
\hline & $\mathrm{OR}^{\mathrm{c}}$ & $\mathrm{LI}^{\mathrm{C}}$ & $\mathrm{LS}^{\mathrm{c}}$ & $E D^{c}$ & OR & LI & LS & ED \\
\hline Intercepto & 0,07 & 0,06 & 0,09 & 1,23 & 0,09 & 0,07 & 0,10 & 1,20 \\
\hline 15 a 24 anos & 0,86 & 0,75 & 0,98 & 1,20 & 1,47 & 1,32 & 1,63 & 1,20 \\
\hline 25 a 49 anos & 1,05 & 0,90 & 1,22 & 1,20 & 1,89 & 1,68 & 2,12 & 1,24 \\
\hline 50 a 64 anos & 1,47 & 1,24 & 1,75 & 1,24 & 2,32 & 2,03 & 2,65 & 1,23 \\
\hline Auto consumo & 1,31 & 0,88 & 1,95 & 1,25 & 1,09 & 0,88 & 1,34 & 1,22 \\
\hline Empregador & 0,89 & 0,72 & 1,10 & 1,27 & 1,11 & 0,92 & 1,33 & 1,24 \\
\hline Empregado sem carteira & 0,81 & 0,71 & 0,93 & 1,25 & 0,94 & 0,86 & 1,02 & 1,26 \\
\hline Conta própria & 0,77 & 0,68 & 0,88 & 1,18 & 1,05 & 0,96 & 1,15 & 1,22 \\
\hline Desempregado & 0,93 & 0,80 & 1,09 & 1,20 & 1,09 & 0,99 & 1,20 & 1,26 \\
\hline Aposentado & 1,85 & 1,55 & 2,20 & 1,25 & 1,26 & 1,14 & 1,40 & 1,28 \\
\hline Estudante & 0,93 & 0,81 & 1,08 & 1,21 & 0,91 & 0,83 & 1,01 & 1,26 \\
\hline 5 a 8 fundamental & 1,06 & 0,95 & 1,17 & 1,24 & 1,00 & 0,93 & 1,08 & 1,21 \\
\hline 8 a 11 segundo grau & 1,27 & 1,14 & 1,42 & 1,20 & 1,07 & 0,99 & 1,17 & 1,21 \\
\hline superior & 1,69 & 1,49 & 1,91 & 1,24 & 1,26 & 1,15 & 1,38 & 1,25 \\
\hline \multicolumn{9}{|l|}{ Posição do chefe no mercado } \\
\hline Auto consumo & 0,80 & 0,56 & 1,16 & 1,18 & 0,93 & 0,76 & 1,15 & 1,18 \\
\hline Empregador & 1,02 & 0,85 & 1,23 & 1,28 & 0,87 & 0,78 & 0,96 & 1,25 \\
\hline Empregado sem carteira & 0,95 & 0,84 & 1,09 & 1,26 & 0,93 & 0,87 & 1,00 & 1,25 \\
\hline Conta própria & 0,87 & 0,78 & 0,98 & 1,20 & 0,90 & 0,85 & 0,96 & 1,24 \\
\hline Desempregado & 1,09 & 0,92 & 1,31 & 1,23 & 1,06 & 0,95 & 1,19 & 1,24 \\
\hline Aposentado & 0,78 & 0,67 & 0,90 & 1,20 & 0,94 & 0,87 & 1,02 & 1,24 \\
\hline Estudante & 1,47 & 0,85 & 2,55 & 1,32 & 0,92 & 0,64 & 1,32 & 1,18 \\
\hline Afazeres domésticos & 0,97 & 0,84 & 1,13 & 1,23 & 1,01 & 0,92 & 1,11 & 1,26 \\
\hline Outros & 1,12 & 0,85 & 1,50 & 1,39 & 1,02 & 0,85 & 1,23 & 1,23 \\
\hline 2 salários ou mais & 1,33 & 1,21 & 1,46 & 1,24 & 1,39 & 1,30 & 1,49 & 1,23 \\
\hline Tamanho da família & 0,95 & 0,93 & 0,97 & 1,25 & 0,93 & 0,92 & 0,94 & 1,27 \\
\hline
\end{tabular}

a Restrição das atividades nos 15 dias que antecederam o estudo.

b Categorias de referência: idade de 10 a 14 anos; raça não branca; escolaridade sem instrução; trabalhador com carteira assinada; renda de 0 a 0,75 salário mínimo; sexo feminino para chefe de família; idade do chefe entre 45 e 54 anos.

${ }^{c} \mathrm{OR}=$ razão de chances; $\mathrm{LI}$ = limite inferior; $\mathrm{LS}$ = limite superior; ED = efeito do desenho amostral.

serviços na presença de restrição de atividades.

Entre pessoas que não referiram restrição de atividades, as mulheres integrantes de famílias de maior poder aquisitivo, de famílias com chefes com maior escolaridade e de famílias chefiadas por homens apresentaram maior chance de consumo de serviços preventivos e de acompanhamento.
Tem-se como hipótese que a variável "sexo do chefe" esteja expressando fatores que capacitam o acesso aos serviços de saúde, por exemplo maior cobertura por plano de saúde, e, também, aspectos sociais, tais como o fato de que as mulheres chefes de família tendem a ter maiores responsabilidades no cuidado da família e na gestão dos recursos financeiros. Essas responsabilidades poderiam limitar a capacidade dessas mulheres de consumir serviços de saúde em relação às mulheres que pertencem a famílias chefiadas por homens.

A posição da própria mulher no mercado de trabalho não afetou a chance de uso das mulheres economicamente ativas. Entretanto, as mulheres sem inserção no mercado 
de trabalho - aposentadas e donas de casa - apresentaram maiores chances de uso do que aquelas economicamente ativas. Uma possível explicação para esse resultado é que as donas de casa e as aposentadas apresentam piores condições de saúde do que as mulheres economicamente ativas, condições estas que não teriam sido captadas pela variável de morbidade utilizada. Na literatura, estudos em diferentes contextos apontam resultados discordantes. Alguns estudos sugerem que as donas de casa apresentam piores condições de saúde em comparação às mulheres com inserção no mercado de trabalho, enquanto outros apontam resultados no sentido inverso (21-23). Por outro lado, também é possível que as mulheres que não trabalham fora disponham de mais tempo livre para consumir serviços de saúde, já que não estão submetidas a uma dupla jornada de trabalho.

A constatação de que as chances de uso de serviços de saúde são reduzidas quando as mulheres pertencem a famílias chefiadas por patrões e autônomos foi inesperada. Nos homens, tal como nas mulheres, a renda familiar per $\mathrm{ca}$ pita e a escolaridade do chefe tiveram impacto positivo nas chances de uso de serviços. No entanto, contrariamente às mulheres, a própria posição dos homens economicamente ativos no mercado de trabalho apresentou efeito direto nas chances de uso de serviços. Homens empregados no setor informal da economia e autônomos apresentaram as menores chances de uso. Os aposentados apresentaram as maiores chances de uso; entretanto, homens membros de famílias cujo chefe é aposentado apresentaram as menores chances de uso de serviços de saúde. Tal discrepância pode estar indicando que, como categoria individual, a condição de aposentado apreende aspectos de morbidade não apreendidos pelas outras variáveis do modelo, enquanto que, como categoria familiar, estaria apreendendo aspectos de desvantagem socioeconômica. Homens autônomos ou membros de famílias cujo chefe é autônomo mostraram-se em desvantagem no consumo de serviços. É possível que esta desigualdades possa, em parte, ser explicada pela cobertura de planos de saúde, que no Brasil associase positivamente à renda, à posição no mercado de trabalho e ao uso de serviços de saúde $(9,16)$.

Um importante resultado desta pesquisa é o reconhecimento de que os modelos testados explicaram menos as variações no consumo entre os homens, particularmente no caso do grupo sem restrição de atividades por problemas de saúde, sugerindo a necessidade de desenvolver modelos explicativos do uso de serviços de saúde pelos homens. Outro resultado a ser destacado é o fato de que, na ausência de restrição de atividades, a escolaridade do chefe da família mostrou-se mais importante do que a escolaridade do próprio indivíduo, em ambos os sexos.

Parte da variação no uso de serviços de saúde deveu-se às características das famílias. Foi possível inferir, a partir do coeficiente de correlação intraclasse dos modelos hierárquicos, que a família tem grande importância na explicação do consumo de serviços de saúde das pessoas sem restrição (predominantemente a serviços preventivos e de acompanhamento). Embora algumas características das famílias tenham explicado variações individuais, nenhuma das características analisadas neste estudo foi capaz de explicar a variação observada entre as famílias. Pesquisas devem ser desenvolvidas para identificar quais características das famílias explicariam as variações observadas.

O tamanho da família (incluindo crianças) apresentou efeito direto sobre o uso de serviços de homens e mulheres sem restrição e de mulheres com restrição. Assumindo esta variável como proxy da estrutura da família, as famílias menores seriam aquelas compostas por pessoas que já não vivem mais com os filhos ou cônjuge, e as maiores, aquelas compostas de casais morando com seus filhos. É possí- vel que o consumo de serviços pelos filhos tenha impacto positivo sobre o consumo das mães.

O emprego de apenas uma variável de necessidade de saúde (restrição de atividades rotineiras) para ajustar o modelo de uso de serviços de saúde pode resultar em ajuste imperfeito, particularmente porque essa variável apreende de maneira melhor os eventos agudos de saúde. Entretanto, como a restrição de atividades refere-se ao mesmo período de tempo da variável dependente (15 dias anteriores à entre vista), além de análises prévias no mesmo banco de dados terem mostrado ser "restrição de atividades" a variável com maior força de associação com uso de serviços de saúde, considera-se que, no geral, essa variável apreendeu as variações de necessidades entre os gêneros.

Vários estudos discutem diferenças sociais na saúde entre os gêneros (24-26); alguns apontam diferenças nos determinantes das desigualdades entre homens e mulheres, outros não. Porém, são escassos os trabalhos que analisaram diferenças nos determinantes das desigualdades sociais no consumo de serviços de saúde por homens e mulheres. Portanto, o presente estudo foi um exercício pioneiro. Vale ressaltar que os resultados se aplicam apenas no contexto da sociedade e do sistema de saúde brasileiros. Porém, tem-se como hipótese que modelos explicativos das desigualdades no uso de serviços de saúde em outras realidades devem variar entre os gêneros sempre que esta categoria expressar uma relação de subordinação.

Assim, a formulação de políticas voltadas para a redução das desigualdades sociais no consumo de serviços de saúde deve considerar a existência de diferenças no padrão de uso de serviços de saúde por mulheres e homens, além da importância de características das famílias neste consumo. Ressalta-se, portanto, a necessidade de melhor especificação dos modelos de uso com relação às dimensões de gênero e família. 


\section{REFERÊNCIAS}

1. Lambrew JM. Diagnosing disparities in health insurance for women: a prescription for change [site da Internet]. Disponível em: www.cmwf.org/programs/insurance/ lambrew_women_493.pdf. Acessado em 1 de maio de 2002.

2. Verbrugge LM, Wingard DL. Sex differentials in health and mortality. Women Health 1987;12(2):103-145.

3. Arber S, Cooper H. Gender differences in health in later life: the new paradox? Soc Sci Med 1999;48(1):61-76.

4. Verbrugge LM. The twain meet: empirical explanations of sex differences in health and mortality. J Health Soc Behav 1989;30(3): 282-304.

5. Marcus AC, Siegel JM. Sex differences in the use of physician services: a preliminary test of the fixed role hypothesis. J Health Soc Behav 1982;23(Sept):186-197.

6. Goldani AM. O regime demográfico brasileiro nos anos 90. Em: Galvão L, Díaz J, eds. Saúde sexual e reprodutiva no Brasil. São Paulo: Editora Hucitec — Population Council; 1999. p.25-69.

7. Schor E, Starfield B, Stidley C, Hankin J. Family health - utilization and effects of family membership. Med Care 1987; 25(7):616-626.

8. Travassos C. Equity in the use of private hospitals contracted by a compulsory insurance scheme in the city of Rio de Janeiro, Brazil, 1986 [tese de doutoramento]. London: The London School of Economics and Political Science; 1992.
9. Travassos C, Viacava F, Fernandes C, Almeida CM. Desigualdades geográficas e sociais na utilização de serviços de saúde no Brasil. Ciência Saúde Coletiva 2000;5(1):133149.

10. Hulka B, Wheat J. Patterns of utilization: the patient perspective. Med Care 1985;(5):438463.

11. Wennberg JE. On patient need, equity, supplier-induced demand, and the need to assess the outcome of common medical practices. Med Care 1985;23(5):512-520.

12. Doyal L. Gender equity in health: debates and dilemmas. Soc Sci Med 2000;51(6):931-939.

13. Fundação Instituto de Geografia e Estatística (IBGE). Estatísticas da saúde: assistência médico-sanitária 1999. Rio de Janeiro: IBGE; 2000.

14. Fundação Instituto de Geografia e Estatística (IBGE). Pesquisa Nacional por Amostra de Domicílios - 1998. Rio de Janeiro: IBGE; 2000

15. Lee ES, Forthofer RN, Lorimer RJ. Analyzing complex survey data. Londres: Sage Publications Inc; 1989.

16. Viacava F, Travassos CM, Pinheiro RS, Brito A. Projeto: gênero e utilização de serviços de saúde no Brasil, financiado pela OPS, [relatório final de pesquisa]. Rio de Janeiro: Departamento de Informações em Saúde/Centro de Informação Científica e Tecnológica/Fundação Oswaldo Cruz; 2001.

17. Arber $\mathrm{S}$. Integrating nonemployment into research on health inequalities. Int J Health Serv 1996;26(3):445-481.
18. Snijder TAB, Bosker RJ. Multilevel analysis: an introduction to basic and advanced multilevel modeling. London: Savage; 1999.

19. Agresti A. Categorical data analysis. New York: John Wiley \& Sons, NY; 1990

20. Shah BV, Barnwell BG, Bieler GS. SUDAAN software for statistical analysis of correlated data. Versão 7.5. Research Triangle Park, NC: Research Triangle Institute; 1979.

21. Wamala SP, Lynch J, Kaplan GA. Women's exposure to early and later life socioeconomic disadvantage and coronary heart disease risk: the Stockholm female coronary risk study. Int J Epidemiol 2001;30(2):275-284.

22. Waldron I, Herold J. Employment, attitudes toward employment, and women's health. Women Health 1986;11(1):79-98.

23. Mukhopadhyay S. Working status and stress of middle class women of Calcutta. J Biosoc Sci 1989;21(1):109-114.

24. Arber S. Class, paid employment and family roles: making sense of structural disadvantage, gender and health status. Soc Sci Med 1991;32(4):425-436.

25. McDonough P, Williams D, House J, Duncan G. Gender and the socioeconomic gradient in mortality. J Health Soc Behav 1999;40(1):1731.

26. Matthews S, Manor O, Power C. Social inequalities in health: are there gender differences? Soc Sci Med 1999;48(1):49-60.
ABSTRACT

Utilization of health care services in Brazil: gender, family characteristics, and social status
Objective. To investigate the pattern of utilization of health care services by men and by women in Brazil.

Methods. We used data from the Brazilian National Household Sample Survey (Pesquisa Nacional por Amostra de Domicílios) carried out in 1998. Logistic regression models were developed to analyze information concerning men and women who had and who did not have restrictions in their routine activities due to health problems during the 15 days before the survey. The individual variables considered were: labor market position (type of employment), amount of schooling completed, and race. Also analyzed were family-related variables: per capita family income, size of the family, and, for the head of the family, the amount of schooling and labor market position. The two-level models (with family and individual variables) showed an effect from family characteristics, but the variables analyzed did not explain that effect.

Results. Women used health services more often than did men, even after controlling for restrictions in routine activities due to health reasons. The use of health services by men and women was dependent on family income and on the social status of the individual, indicating a pattern of social inequality. In both the group with restrictions in their activities and in the group without such restrictions, the men and women differed from each other in their utilization of health care services. Family variables were more important in explaining the utilization of health services among people without restrictions in their activities.

Conclusions. Policies aimed at reducing inequalities in access to health care services must take into consideration the differences between women and men as well as the importance of family characteristics. It is also important to stress the need to include the dimensions of gender and family in the design of health service utilization models. 